\title{
Transversus Abdominis Plane Blockade as Part of a Multimodal Postoperative Analgesia Plan in Patients Undergoing Radical Cystectomy
}

\author{
Richard S. Matulewicz ${ }^{\mathrm{a}, *}$, Mehul Patel $^{\mathrm{a}}$, Brian J. Jordan ${ }^{\mathrm{a}}$, Jacqueline Morano $^{\mathrm{b}}$, Brendan Frainey ${ }^{\mathrm{a}}$, \\ Yasin Bhanji ${ }^{\mathrm{a}}$, Mahreen Bux ${ }^{\mathrm{a}}$, Antoun Nader ${ }^{\mathrm{b}}$, Shilajit D. Kundu ${ }^{\mathrm{a}}$ and Joshua J. Meeks ${ }^{\mathrm{a}}$ \\ ${ }^{a}$ Department of Urology, Northwestern University, Feinberg School of Medicine, Chicago, IL, USA \\ ${ }^{\mathrm{b}}$ Department of Anesthesia, Northwestern University, Feinberg School of Medicine, Chicago, IL, USA
}

\begin{abstract}
.
Background: Radical cystectomy (RC) is a morbid procedure with frequent complications that may benefit from implementation of an enhanced recovery after surgery (ERAS) protocol.

Objective: To examine the benefits of a multimodal analgesic plan that uses continuous transversus abdominis plane (TAP) blockade as part of an ERAS protocol after RC.

Methods: A retrospective comparison of consecutive patients undergoing RC over a 4-year period was conducted. Patients were designated as having surgery either before or after implementation of an ERAS protocol. A major component of the ERAS protocol was a multi-modal analgesia plan with TAP blockade. Patient demographics, comorbidities, operative details, and outcomes, including days to flatus, bowel movement (BM), narcotic usage, and length of stay (LOS) were compared.

Results: In total, 171 patients were included: 100 pre-ERAS and 71 ERAS. There were no differences in age, smoking status, operative approach, or diversion type. The patients in the ERAS cohort were less likely to be male, had a higher median BMI, and more likely to have received neoadjuvant chemotherapy. Total and early postoperative narcotic use were lower in the ERAS cohort: 89 vs. $336 \mathrm{mg}(p<0.001)$ and $62 \mathrm{vs} 203 \mathrm{mg}(p=0.001)$, respectively. The ERAS cohort had fewer days to flatus (3 vs. $4, p<0.001)$ and fewer days to bowel movement (4 vs. $5, p<0.001)$. Median LOS was shorter in the ERAS cohort ( 7 vs. $8.5 \mathrm{~d}, p=0.001$ ). There were no differences in complications or readmission rates between the two cohorts.

Conclusions: TAP blockade as part of an ERAS multi-modal pain plan is associated with low narcotic usage, and significant improvement in time to flatus, BM, and LOS compared to traditional post-RC pain management.
\end{abstract}

Keywords: Regional anesthesia, radical cystectomy, TAP block, ERAS

\section{INTRODUCTION}

Radical cystectomy with pelvic lymph node dissection $(\mathrm{RC})$ is the gold standard treatment for muscle

\footnotetext{
*Correspondence to: Richard S. Matulewicz, MS, MD, Department of Urology, Northwestern University, Feinberg School of Medicine, 675 North St. Clair Street, Suite 20-150, Chicago, IL 60611, USA. Tel.: +1 312308 3238; Fax: +1 312908 7275; E-mail: Richard.matulewicz@northwestern.edu.
}

invasive bladder cancer and is associated with a high risk of complications [1]. Postoperative ileus, resulting from a combination of general anesthesia, bowel manipulation, and opioid pain medications is one of the most common complications associated with RC and is frequently the cause of prolonged length of stay (LOS) [2]. Opioid use increases the inhibitory neural input to the bowel, which disrupts gastrointestinal motility and secretion, leading to hard dry 
stools, straining, incomplete evacuation, abdominal distention, bloating, and reflux [3].

At our institution, we implemented an enhanced recovery after surgery (ERAS) protocol in an effort to standardize care, decrease LOS, and improve outcomes after RC. Similar to many other protocols $[4,5]$, we used several proactive interventions in the perioperative period aimed specifically at avoiding narcotic pain medications and reducing the risk of post-operative ileus. One of the critical components was the use of regional anesthesia with transversus abdominis plane (TAP) catheters in additional to other non-narcotic pain management. We sought to examine the benefits of this multimodal analgesic plan within our ERAS protocol.

Regional anesthesia offers an alternate treatment for post-operative pain and play an integral role in the reduction of opioid usage. TAP catheters block the neural afferents of the anterolateral abdominal wall in the dermatomes T6-L1 [6, 7]. Such nerves located within this plane include the intercostal, subcostal, illioinguinal, and iliohypogastric which are within the area of the surgical incision and are thought to be a significant source of post-operative pain.

We hypothesize that implementation of an opioidsparing pain management plan in RC patients would decrease the incidence of post-operative ileus and speed return of bowel function and decrease LOS. It was our goal to reduce median length of stay to less than or equal to 7 days as this was considered a surrogate marker of good postoperative outcomes. In this study, we evaluate opioid and ketorolac usage, return of bowel function, and LOS in a population of $\mathrm{RC}$ patients prior to and after the implementation of an ERAS protocol at our institution.

\section{MATERIALS AND METHODS}

\section{Study population}

A retrospective review of patients undergoing $\mathrm{RC}$ from April 2011 to October 2016 was conducted. Patients were separated into two cohorts, pre-ERAS and ERAS. Regional anesthesia became universally used for patients undergoing $\mathrm{RC}$ at our institution in November 2014 as part of the implementation of our ERAS protocol. All patients received general anesthesia and intraoperative pain management was left to the discretion of the anesthesia providers. TAPs were chosen in order to avoid the significant side effects of epidural analgesia (EA) such as epidural hematoma, increased hypotension, and excessive intravenous fluid administration [8]. Bilateral TAP catheters were placed by the anesthesia team preoperatively or immediately post-operatively, and in rare cases on post-operative day (POD) 1 . The acute pain service evaluated the patients in the recovery room and on day 1, 2 and 3 and additional boluses of $0.1 \%$ ropivacaine through the catheter were given for pain score greater than 3 on the visual analogue pain scale. The TAP catheters were removed on POD 3 per protocol.

The ERAS cohort patients were also managed with around the clock standing acetaminophen and ketorolac (when renal function allowed). Narcotic use was limited to severe refractory pain. Other pertinent features of our ERAS protocol included avoidance of bowel preparation preoperatively, no routine use of postoperative nasogastric (NG) tube, and early feeding (low volume clear liquids on POD 1) as well as early ambulation. The commonly used medication alvimopan was not available at our institution and was therefore not used.

During the pre-ERAS era, neither postoperative management nor pain management were standardized. Pain control was achieved using any combination of opioid patient controlled analgesia (PCA), combined opioid/local anesthetic patient controlled epidural analgesia (PCEA), as well as narcotics, acetaminophen, and ketorolac on an as needed basis.

\section{Data collection}

Patient information including sex, age, smoking status, BMI, associated medical comorbidities, and receipt of neoadjuvant chemotherapy was collected via retrospective chart review. Pertinent operative details such as surgical approach (open vs. robotic), urinary diversion type (ileal conduit vs. continent diversion- either neobladder or catheterizeable pouch), and operative time were also collected. Narcotic requirements were aggregated for each patient and converted to oral morphine equivalents (in milligram) using standard opioid conversions [9, 10]. Narcotic use was assessed in sum total for each patient's hospital stay and during the "early postoperative period" defined as POD 0 through POD 3. Opioids used during induction or maintenance of general anesthesia were excluded from calculations. Ketorolac usage was measured by the number of patients that received any single dose and mean milligrams used. LOS was determined and return of bowel function was assessed by time to first flatus 
and time to initial bowel movement (in days). Potential gastrointestinal complications were considered by assessing need for postoperative "rescue" NG tube placement or need for reoperation within 30 days. NG tubes were placed for refractory nausea and vomiting or prolonged ileus. In addition, 30-day and 90-day readmission rates as well as 90 -day mortality rates were collected for each group (evaluation limited to Emergency Department visits and direct hospital admissions at our institution).

\section{Statistical analysis}

Statistical analysis was performed with STATA v13. Median days to flatus, bowel movement, LOS, and narcotic usage (oral morphine equivalents in $\mathrm{mg}$ ) were compared using the Mann-Whitney Test. Mean ketorolac usage was compared using the $t$-test. Categorical variables were compared using a chi-squared test. A binomial logistic regression model was constructed to examine the relationship between care era (pre-ERAS vs. ERAS) and hospital LOS less than or equal to seven days adjusting for age, gender, obesity, several medical comorbidities (hypertension, diabetes, heart disease, lung disease, kidney disease, history of venous thromboembolism), smoking status, receipt of neoadjuvant chemotherapy, operative approach, diversion type, and operative duration. A LOS of seven or fewer days was chosen a priori during development of our ERAS protocol as an institutional goal based on review of multicenter literature and as an acceptable composite metric of a typical uncomplicated postoperative course.

\section{RESULTS}

In total, 171 consecutive patients who underwent RC were included: 100 patients in the pre-ERAS cohort and 71 in the ERAS cohort. There were no differences in age, smoking status, operative approach (open vs. robotic), or urinary diversion type between the two cohorts (Table 1). The ERAS cohort had a lower percentage of male patients $(69 \%$ vs. $83 \%$, $p=0.03$ ) and more patients who received neoadjuvant chemotherapy ( $38 \%$ vs. $21 \%, p=0.02)$. In terms of comorbidities, the ERAS cohort had a significantly higher average BMI (28.6 vs $26.3, p=0.01$ ) and history of venous thromboembolism (VTE) prior to surgery $(15.5 \%$ vs $5.0 \%, p=0.02)$, but otherwise had similar rates of hypertension, diabetes, and cardiac or pulmonary comorbidities. Median operative time was significantly shorter (356 vs. 434 minutes,

Table 1

Comparison of patient demographics including age, sex, smoking status, comorbidities, neoadjuvant chemotherapy, operative approach, and urinary diversion in the pre-ERAS and ERAS cohorts

\begin{tabular}{|c|c|c|c|}
\hline Demographics & Pre-ERAS $(n=100)$ & ERAS $(n=71)$ & $\overline{p \text {-value }}$ \\
\hline Age [IQR] & $68.9[63.7-77.7]$ & $71.5[62.6-78.3]$ & 0.59 \\
\hline Male Sex $(\%, n)$ & $83.0 \%(83)$ & $69.0 \%(49)$ & 0.03 \\
\hline \multicolumn{4}{|l|}{ Comorbidities } \\
\hline Median BMI [IQR] & $26.3[23.1-29.9]$ & $28.6[25.3-32.2]$ & 0.01 \\
\hline Obese $(\%, \mathrm{n})$ & $24.0 \%(24)$ & $36.6 \%(26)$ & 0.07 \\
\hline Hypertension $(\%, \mathrm{n})$ & $58.0 \%(58)$ & $52.1 \%(37)$ & 0.45 \\
\hline Heart Problems $(\%, \mathrm{n})$ & $23.0 \%(23)$ & $25.4 \%(18)$ & 0.72 \\
\hline Diabetes Mellitus $(\%, \mathrm{n})$ & $22.0 \%(22)$ & $15.5 \%(11)$ & 0.29 \\
\hline Lung Disease $(\%, \mathrm{n})$ & $9.0 \%(9)$ & $16.9 \%(12)$ & 0.12 \\
\hline Kidney Disease $(\%, \mathrm{n})$ & $11.0 \%(11)$ & $8.5 \%(6)$ & 0.58 \\
\hline History of VTE $(\%, \mathrm{n})$ & $5.0 \%(5)$ & $15.5 \%(11)$ & 0.02 \\
\hline \multicolumn{4}{|l|}{ Smoker } \\
\hline Never $(\%, \mathrm{n})$ & $22.0 \%(22)$ & $31.0 \%(22)$ & 0.38 \\
\hline Former $(\%, \mathrm{n})$ & $63.0 \%(63)$ & $57.8 \%(41)$ & \\
\hline Current $(\%, \mathrm{n})$ & $15.0 \%(15)$ & $11.2 \%(8)$ & \\
\hline \multicolumn{4}{|l|}{ Neoadjuvant Chemotherapy } \\
\hline Given $(\%, \mathrm{n})$ & $21.0 \%(21)$ & $38.0 \%(27)$ & 0.02 \\
\hline \multicolumn{4}{|l|}{ Operative Approach } \\
\hline Open $(\%, \mathrm{n})$ & $79.0 \%(79)$ & $81.7 \%(58)$ & 0.66 \\
\hline Robotic $(\%, \mathrm{n})$ & $21.0 \%(21)$ & $18.3 \%(13)$ & \\
\hline \multicolumn{4}{|l|}{ Diversion } \\
\hline Ileal Conduit $(\%, \mathrm{n})$ & $69.1 \%(67)$ & $76.1 \%(54)$ & 0.32 \\
\hline Continent (IP/NB) $(\%, n)$ & $30.9 \%(30)$ & $23.9 \%(17)$ & \\
\hline \multicolumn{4}{|l|}{ Operative Time } \\
\hline Median minutes [IQR] & $434[338.5-522]$ & $356[287-441]$ & $<0.01$ \\
\hline
\end{tabular}


Table 2

Comparison of perioperative outcomes, narcotic usage, and GI recovery in the pre-ERAS and ERAS cohorts

\begin{tabular}{lccc}
\hline & pre-ERAS $(n=100)$ & ERAS $(n=71)$ & $p$-value \\
\hline Median LOS (days) [IQR] & $8.5[7-12]$ & $7[5-11]$ & $<0.01$ \\
LOS $\leq 7$ days (\%, $\mathrm{n})$ & $34.0 \%(34)$ & $59.2 \%(42)$ & $<0.01$ \\
Primary Pain Management & & & $<0.01$ \\
$\quad$ TAPS & $0.0 \%(0)$ & $100 \%(71)$ & \\
PCEA/epidural & $23.0 \%(23)$ & $0.0 \%(0)$ & \\
PCA & $59.0 \%(59)$ & $0.0 \%(0)$ & $<0.001$ \\
Ketorolac Used (>0 mg) & $30 \%(30)$ & $78.9 \%(56)$ & $<0.001$ \\
Ketorolac Used (mean mg, SD) & $40.4(88.5)$ & $177.2(179.8)$ & \\
Narcotic Usage (oral mg morphine) & & & $<0.01$ \\
Overall (entire hospital stay) & $336[93-678]$ & $89[0-178]$ & $<0.01$ \\
POD 0-3 & $203[38-418]$ & $62[24-154]$ & $<0.01$ \\
Time to Flatus - Days [IQR] & $4[4-6]$ & $3[2-4]$ & 0.09 \\
Time to BM - Days [IQR] & $5[4-7]$ & $4[3-6]$ & 0.81 \\
"Rescue" NG Tube Placement & $16.0 \%(16)$ & $26.8 \%(19)$ & 0.13 \\
30 Day Reoperation & $5.0 \%(5)$ & $11.2 \%(3)$ & 0.20 \\
30 Day Readmission & $20.0 \%(20)$ & $29.6 \%(21)$ & 0.68 \\
90 Day Readmission & $39.0 \%(39)$ & $2.8 \%(2)$ & \\
90 Day Mortality & $4.0 \%(4)$ & & \\
\hline
\end{tabular}

$p=0.002)$ in the ERAS cohort. Anesthesia time was not affected by TAP catheter placement as this was performed in the preoperative holding area or PACU and not within the operating room.

Total and early (POD 0-3) narcotic use were significantly lower in the ERAS cohort: 89 vs. $336 \mathrm{mg}$ $(p<0.01)$ and 62 vs $203 \mathrm{mg}(p<0.01)$, respectively. Any usage of ketorolac was significantly higher in the ERAS cohort: $78.9 \%$ vs. $30 \%(p<0.001)$ as was total usage $(177.2 \mathrm{mg}$ vs. $40.4 \mathrm{mg}(p<0.001))$. The ERAS cohort had better GI recovery with fewer days to flatus ( 3 vs $4, p<0.01$ ) and fewer days to bowel movement ( 4 vs. $5, p<0.01$ ) (Table 2 ). In addition, the median
LOS was significantly shorter in the ERAS cohort ( 7 vs. $8.5 \mathrm{~d}, p<0.01$ ), with a significantly higher percentage of patients with $\operatorname{LOS} \leq 7 \mathrm{~d}(59.2 \%$ vs $34 \%$, $p<0.01)$. There was no difference in need for rescue NG tube, reoperation, 30-day/90-day readmission, or 90-day mortality among the two groups. There were no specific complications related to placement or use of TAP catheters in any patients.

When adjusting for numerous perioperative and patient factors, having surgery during the ERAS era was associated with a higher likelihood of LOS less than or equal to 7 days (OR 2.51, 95\% CI 1.18-5.34) (Table 3). Additionally, being a former (OR 0.43,

Table 3

Binomial logistic regression model examining factors associated with length of stay less than or equal to 7 days adjusting for patient demographics, comorbidities, and peri-operative details

\begin{tabular}{lccc}
\hline & Odds Ratio & 95\% Confidence Interval & $p$-value \\
\hline ERAS vs. pre-ERAS era & 2.51 & $1.18-5.34$ & 0.02 \\
Age (+1 year) & 1.02 & $0.98-1.06$ & 0.40 \\
Male Sex vs. Female & 1.66 & $0.70-3.93$ & 0.25 \\
Obesity & 0.76 & $0.33-1.75$ & 0.53 \\
Hypertension & 0.62 & $0.31-1.28$ & 0.20 \\
Heart Disease & 0.49 & $0.20-1.20$ & 0.12 \\
Diabetes Mellitus & 0.62 & $0.25-1.57$ & 0.32 \\
Lung Disease & 0.69 & $0.22-2.15$ & 0.52 \\
Kidney Disease & 0.77 & $0.20-2.98$ & 0.70 \\
VTE & 2.91 & $0.79-10.68$ & 0.11 \\
Smoker (vs. non-smoker) & & & \\
$\quad$ Former & 0.43 & $0.19-0.98$ & $0.05^{*}$ \\
Current & 0.25 & $0.07-0.87$ & 0.03 \\
Neoadjuvant Chemotherapy & 1.92 & $0.86-4.28$ & 0.11 \\
Robotic Approach & 2.45 & $0.85-7.11$ & 0.10 \\
Continent Diversion Type & 1.43 & $0.55-3.70$ & 0.47 \\
Operative Time (each additional 15 mins) & 0.97 & $0.93-1.03$ & 0.32 \\
\hline
\end{tabular}

$* p$-value $=0.045$ prior to rounding. 
95\% CI 0.19-0.98) or current smoker (OR 0.25, 95\% CI 0.07-0.87) was associated with LOS greater than 7 days compared to never smokers.

\section{DISCUSSION}

Radical cystectomy is a complex extirpative procedure commonly performed on elderly patients with irreversible comorbidities. In order to continue to improve outcomes, urologists must optimize known, modifiable factors in the setting of these static hurdles. Systemic narcotic usage for postoperative pain control should no longer be considered a first line option in RC patients considering their known short and long term side effects. We report the efficacy of a multimodal analgesic plan featuring local anesthetic continuous infusion TAP catheters with scheduled acetaminophen and ketorolac as able. This pain management plan was safely associated with patients receiving significantly lower doses of narcotics and resulted in improved convalescence in the form of hastened GI recovery and shorter lengths of stay in $\mathrm{RC}$ patients.

The use of TAP catheters or TAP blocks postoperatively has been previously explored in other abdominal surgeries. Randomized controlled trials in radical retropubic prostatectomy, total abdominal hysterectomy, and other major abdominal procedures generally resulted in lower pain scores and opioid usage without obvious widespread benefits in GI recovery or length of stay $[6,11,12]$. Since many of these procedures are followed by short hospital stays and do not include significant bowel manipulation, the greater efficacy seen in RC in our study may be inherent to the complexity of the procedure and prolonged postoperative course in RC patients.

Efforts to mitigate the downstream effects of narcotics by avoiding them entirely represent one means of optimizing bowel function. We achieved this goal through a variety of measures. We significantly increased ketorolac usage from $30 \%$ pre-ERAS to $78.9 \%$ in the ERAS cohort $(p<0.001)$. Additional alternatives have been explored and include traditional means of non-narcotic pain control such as epidural analgesia (EA) and novel agents such as alvimopan, a selective peripheral mu-opioid receptor antagonist which prevents opioid related side effects on the gastrointestinal tract. A recent meta-analysis of TAP blocks showed comparable pain control to epidural analgesia (EA) [13], and several smaller studies have demonstrated less hypotension in the TAP groups [14-16]. It has been shown that TAP catheter infusions deliver greater spread of the local anesthetic in the distribution of the lower intercostal nerves $(\mathrm{T} 10,11,12)$ and $\mathrm{L} 1$ which can provide excellent analgesia after lower abdominal incisions [17].

Alternatively, the efficacy of alvimopan has been established in multiple clinical trials for open abdominal surgery [18]. Specifically for bladder cancer patients, a multicenter randomized controlled trial of alvimopan vs. placebo in patients undergoing RC showed a shorter mean time to first bowel movement and toleration of solid food (5.5 vs 6.8 days, HR 1.77; 95\% CI 1.4-2.3; $p<0.0001)$ [19]. In centers where alvimopan is unavailable such as in the UK [5], TAP catheters may represent a suitable alternative though comparative efficacy and cost comparisons between the two must be further explored. An additional consideration for TAPs is the necessity of skilled regional anesthesiologists for TAP placement, though intraoperative surgeon single-shot blockade at the conclusion of the case has been reported. Additionally, the results of our particular ERAS protocol that did not use alvimopan should be interpreted accordingly to other protocols that did use alvimopan.

Though most likely related to improved GI recovery, in our study, postoperative LOS was decreased from a median of 8.5 to 7.0 days in the ERAS cohort without a significant increase in readmission rates or mortality. This finding is consistent with our hypothesis and prior data that implicates postoperative ileus as a major contributor to increased complications and therefore LOS. The use of "rescue" NG tubes for nausea/vomiting or ileus after surgery was surprisingly similar between the two groups however. One hypothesis is that ileus is an unavoidable inevitability in certain patients and ERAS protocols get patients to their destined short term clinical end points-both good and bad, faster. This may explain how the LOS was decreased in the ERAS group with no change in the percentage of patients who required NG tube placement for ileus. While there has been concern regarding the safety of shorter LOS and how it relates to a greater risk of "at home" complications or readmissions, our findings are consistent with previous studies [20, 21] that have demonstrated shorter LOS to be safe, but this issue must continue to be explored.

In addition to improving return of bowel function, our study also demonstrated a significant reduction in the necessity of narcotic pain medications during patients' entire hospital stay and particularly in the 
early (POD 0-3) postoperative course. Opioid abuse has increased substantially over time and various initiatives have been made to combat the epidemic $[22,23]$. Studies have shown that a small percentage of opioid naïve patients continue to use opioids long after surgery, contributing to the number of chronic opioid users [24-26]. Also of note, current smoking status was associated with an extended length of stay after adjusting for several patient comorbidities even in the ERAS cohort. This reinforces the findings of previous studies that smoking cessation prior to RC can improve both early recovery and long-term oncologic outcomes [27, 28].

There are a number of limitations in this study. Due to its retrospective nature and lack of consistent post-operative pathway prior to the implementation of the ERAS protocol, there are numerous factors that we are unable to control for. Though two comparative cohorts were well matched with regard to comorbidities, demographics, operative approach, and diversion type, the operative time and number of patients who received neoadjuvant chemotherapy were different, which may have biased the results. The ERAS cohort had shorter operative times by nearly an hour. This may represent improvements on the surgical learning curve among included surgeons. Given the large number of individual surgeons included and the resulting heterogeneity of the surgeon cohort, we were unable to adjust for learning curve. Longer operative times have been associated with increased complications rates which lead to prolonged hospital stay [1, 2, 29] and our findings must be interpreted with this in mind. Additionally, the phenomenon of increased operative volume and familiarity with procedures improving outcomes should also be recognized from the non-surgical ancillary staff perspective as well.

Most importantly, while our ERAS protocol's main theme has been opioid avoidance, our protocol did include additional interventions such as eschewing routine bowel preparation, avoiding routine use of postoperative NG tube, and attempting earlier feeding which may have contributed to the outcomes seen in this study and other studies. However, as part of a multimodal post-operative pain plan, TAP catheter blockade was associated with a significant improvement in GI recovery. These findings warrant further exploration and validation, especially when compared to other techniques or medications. TAP catheters should be considered for study in a controlled prospective multicenter manner.

\section{CONCLUSIONS}

TAP blockade as part of a multimodal postoperative pain plan is safe and associated with low narcotic usage, and significant improvement in time to flatus, BM, and LOS compared to traditional post-RC management.

\section{CONFLICT OF INTEREST}

The authors have no conflict of interest to report.

\section{DISCLOSURES}

This work was funded, in part, by grant support provided by the Alumni Association of Northwestern University. No financial conflict of interests exist otherwise.

\section{REFERENCES}

[1] Pietzak EJ, Hwang WT, Malkowicz SB, Guzzo TJ. Factors influencing the length of stay after radical cystectomy: Implications for cancer care and perioperative management. Ann Surg Oncol 2014;21(13):4383-9.

[2] Chang SS, Baumgartner RG, Wells N, Cookson MS, Smith Jr JA. Causes of increased hospital stay after radical cystectomy in a clinical pathway setting. The Journal of Urology. 2002;167(1):208-11.

[3] Panchal SJ, Muller-Schwefe P, Wurzelmann JI. Opioidinduced bowel dysfunction: Prevalence, pathophysiology and burden. Int J Clin Pract. 2007;61(7):1181-7.

[4] Maloney I, Parker DC, Cookson MS, Patel S. Bladder cancer recovery pathways: A systematic review. Bladder Cancer. 2017;3(4):269-81.

[5] Pang KH, Groves R, Venugopal S, Noon AP, Catto JWF. Prospective implementation of enhanced recovery after surgery protocols to radical cystectomy. European Urology. 2017.

[6] McDonnell JG, O’Donnell B, Curley G, Heffernan A, Power C, Laffey JG. The analgesic efficacy of transversus abdominis plane block after abdominal surgery: A prospective randomized controlled trial. Anesthesia and Analgesia. 2007;104(1):193-7.

[7] Petersen PL, Hilsted KL, Dahl JB, Mathiesen O. Bilateral transversus abdominis plane (TAP) block with 24 hours ropivacaine infusion via TAP catheters: A randomized trial in healthy volunteers. BMC Anesthesiol. 2013;13(1):30.

[8] Hughes MJ, Ventham NT, McNally S, Harrison E, Wigmore S. Analgesia after open abdominal surgery in the setting of enhanced recovery surgery: A systematic review and meta-analysis. JAMA Surg. 2014;149(12):1224-30.

[9] Doyle D. Oxford textbook of palliative medicine. 3rd ed. Oxford; New York: Oxford University Press; 2004.

[10] Porter MP, Lin DW. Trends in renal cancer surgery and patient provider characteristics associated with partial nephrectomy in the United States. Urologic Oncology. 2007;25(4):298-302. 
[11] Carney J, McDonnell JG, Ochana A, Bhinder R, Laffey JG. The transversus abdominis plane block provides effective postoperative analgesia in patients undergoing total abdominal hysterectomy. Anesthesia and Analgesia. 2008;107(6):2056-60.

[12] Elkassabany N, Ahmed M, Malkowicz SB, Heitjan DF, Isserman JA, Ochroch EA. Comparison between the analgesic efficacy of transversus abdominis plane (TAP) block and placebo in open retropubic radical prostatectomy: A prospective, randomized, double-blinded study. J Clin Anesth. 2013;25(6):459-65.

[13] Ma N, Duncan JK, Scarfe AJ, Schuhmann S, Cameron AL. Clinical safety and effectiveness of transversus abdominis plane (TAP) block in post-operative analgesia: A systematic review and meta-analysis. J Anesth. 2017;31:432-52.

[14] Ganapathy S, Sondekoppam RV, Terlecki M, Brookes J, Adhikary SD, Subramanian L. Comparison of efficacy and safety of lateral-to-medial continuous transversus abdominis plane block with thoracic epidural analgesia in patients undergoing abdominal surgery: A randomised, open-label feasibility study. Eur J Anaesthesiol. 2015;32:797-804.

[15] Rao Kadam V, Van Wijk RM, Moran JI, Miller D. Epidural versus continuous transversus abdominis plane catheter technique for postoperative analgesia after abdominal surgery. Anaesthesia and Intensive Care. 2013;41(4): 476-81.

[16] Wahba SS, Kamal SM. Analgesic efficacy and outcome of transversus-abdominis plane block versus low thoracicepidural analgesia after laparotomy in ischemic heart disease patients. J Anesth. 2014;28:517-23.

[17] Carney J, Finnerty O, Rauf J, Bergin D, Laffey JG, Mc Donnell JG. Studies on the spread of local anaesthetic solution in transversus abdominis plane blocks. Anaesthesia. 2011;66(11):1023-30.

[18] Matulewicz RS, Brennan J, Pruthi RS, Kundu SD, Gonzalez CM, Meeks JJ. Radical cystectomy perioperative care redesign. Urology. 2015;86(6):1076-86.

[19] Lee CT, Chang SS, Kamat AM, Amiel G, Beard TL, Fergany A, et al. Alvimopan accelerates gastrointestinal recovery after radical cystectomy: A multicenter randomized placebo-controlled trial. European Urology. 2014;66(2):265-72.
[20] Altobelli E, Buscarini M, Gill HS, Skinner EC. Readmission rate and causes at 90 -day after radical cystectomy in patients on early recovery after surgery protocol. Bladder Cancer. 2017;3(1):51-6.

[21] Djaladat H, Katebian B, Bazargani ST, Miranda G, Cai J, Schuckman AK, et al. 90-Day complication rate in patients undergoing radical cystectomy with enhanced recovery protocol: A prospective cohort study. World Journal of Urology. 2016.

[22] Gellad WF, Good CB, Shulkin DJ. Addressing the opioid epidemic in the United States: Lessons from the department of veterans affairs. JAMA Intern Med. 2017.

[23] Penm J, MacKinnon NJ, Boone JM, Ciaccia A, McNamee C, Winstanley EL. Strategies and policies to address the opioid epidemic: A case study of Ohio. J Am Pharm Assoc (2003). 2017;57(2S):S148-S53.

[24] Alam A, Gomes T, Zheng H, Mamdani MM, Juurlink DN, Bell CM. Long-term analgesic use after low-risk surgery: A retrospective cohort study. Archives of Internal Medicine. 2012;172(5):425-30.

[25] Carroll I, Barelka P, Wang CK, Wang BM, Gillespie MJ, McCue R, et al. A pilot cohort study of the determinants of longitudinal opioid use after surgery. Anesthesia and Analgesia. 2012;115(3):694-702.

[26] Steyaert A, Lavand'homme P. Postoperative opioids: Let us take responsibility for the possible consequences. Eur J Anaesthesiol. 2013;30(2):50-2.

[27] Kim PH, Kent M, Zhao P, Sfakianos JP, Bajorin DF, Bochner $\mathrm{BH}$, et al. The impact of smoking on pathologic response to neoadjuvant cisplatin-based chemotherapy in patients with muscle-invasive bladder cancer. World Journal of Urology. 2014;32(2):453-9.

[28] Crivelli JJ, Xylinas E, Kluth LA, Rieken M, Rink M, Shariat SF. Effect of smoking on outcomes of urothelial carcinoma: A systematic review of the literature. European Urology. 2014;65(4):742-54.

[29] Lavallee LT, Schramm D, Witiuk K, Mallick R, Fergusson $\mathrm{D}$, Morash C, et al. Peri-operative morbidity associated with radical cystectomy in a multicenter database of community and academic hospitals. PLoS One. 2014;9(10):e111281. 